\title{
Meshing Force of Misaligned Spline Coupling and the Influence on Rotor System
}

\author{
Guang Zhao, Zhansheng Liu, and Feng Chen \\ School of Energy Science and Engineering, Harbin Institute of Technology, Harbin 150001, China \\ Correspondence should be addressed to Guang Zhao, zghitboy@hotmail.com
}

Received 4 January 2008; Accepted 24 March 2008

Recommended by Kazuhiko Kawaike

\begin{abstract}
Meshing force of misaligned spline coupling is derived, dynamic equation of rotor-spline coupling system is established based on finite element analysis, the influence of meshing force on rotor-spline coupling system is simulated by numerical integral method. According to the theoretical analysis, meshing force of spline coupling is related to coupling parameters, misalignment, transmitting torque, static misalignment, dynamic vibration displacement, and so on. The meshing force increases nonlinearly with increasing the spline thickness and static misalignment or decreasing alignment meshing distance (AMD). Stiffness of coupling relates to dynamic vibration displacement, and static misalignment is not a constant. Dynamic behaviors of rotor-spline coupling system reveal the following: $1 \mathrm{X}$-rotating speed is the main response frequency of system when there is no misalignment; while 2X-rotating speed appears when misalignment is present. Moreover, when misalignment increases, vibration of the system gets intricate; shaft orbit departs from origin, and magnitudes of all frequencies increase. Research results can provide important criterions on both optimization design of spline coupling and trouble shooting of rotor systems.
\end{abstract}

Copyright $\odot 2008$ Guang Zhao et al. This is an open access article distributed under the Creative Commons Attribution License, which permits unrestricted use, distribution, and reproduction in any medium, provided the original work is properly cited.

\section{INTRODUCTION}

The problem of misalignment encountered in rotating machinery is of great concern to designers and maintenance engineers. The need of understanding the phenomena and its principle is important to practical engineers for the purpose of trouble shooting. Most rotating machinery consists of a driver and a driven machine coupled through some type of couplings [1]. There are many types of industrial couplings one of which is spline coupling. Spline coupling allows shaft to be easily dismantled while providing a high-torque capacity for minimum size. In addition, it can allow relative axial and radial motions between the coupled shafts [2]. For these reasons, spline coupling is one of the most commonly used couplings to link oxidizer pump and reducing agent pump in rocket engine, aviation engine and generator [3], reducer and output shaft in large ship, and so on. However, because of high-rotating speed (such as pump in rocket) or large transmitting torque (such as output shaft in ship) of rotors connected by spline coupling, the support conditions which get much rigorous would cause misalignment of spline coupling unavoidablely. It has been observed by the author on several occasions that rotating machinery stability conditions can change should the alignment state between the driver and the driven machines changes [4]. In one side, angular misalignment can greatly increase the slip [2], relative slip of small amplitude can occur between the contacting tooth surfaces. This can give rise to fretting damage which may limit the life of the coupling and has the potential to compromise integrity. This has been a particular concern in aircraft mechanical systems and has been studied experimentally for many years $[5,6]$. In another side, misalignment can causes high vibrations with different symptoms that sometimes cannot be explained [7-9].

There are many discussions in the industry regarding the stiffness, damping, and wear of spline coupling. Marmol et al. [10] developed a mathematical model to predict the lateral and angular stiffness and damping coefficients of spline couplings. Based on these coefficients, they calculated the natural frequencies of a rotor system and compared them to the experimental data. With Marmol's linear stiffness and damping coefficients, rotor-spline coupling-bearing system was studied by Park [11]. In the design charts of flanged and curvic couplings provided by Bannister [12], flexural 
stiffness values were provided. In 1994, the report of NASA [13] provides the first opportunity to quantify the angular stiffness and equivalent viscous damping coefficients of an axial-spline coupling used in high-speed turbomachinery with experimental method. Ku et al. [14] developed experimental research on dynamic coefficient of spline coupling. Peng and Li [15] considered the bending, shear, and compress deformation when calculating the load capability of involute spline, while the deformation of elastic foundation was neglected. Walton et al. [16] developed three nonlinear analytical models representing interference fits and axial and curvic spline couplings. The results show the predictability of rotor system instability due to internal fiction, which has been experimentally observed, especially for axial splines.

Summarizing the above discussions, many researchers pay attention to the stiffness of spline coupling, experiment is an effectual and costly way. However, because of misalignment, it is difficult to obtain the accurate results. In addition, spline coupling, which is one element of rotor system essentially, is separated from its rotor system, there is not enough academic research that integrates the spline stiffness, wear, and misalignment with the rotor system. Consequently, inclusive and principled understanding of the roots of spline wear and misalignment is difficult to achieve.

The present work studied the elastic deformation of single spline referring to the analysis method of tooth deformation; meshing force of misaligned spline coupling was derived according to the deformation configuration relationships of all splines in coupling. Graphical simulations were actualized to obtain the influence of misalignment and structure parameters on meshing force. To gain a greater insight of the effect of spline coupling on rotor system, the dynamic model of rotor-spline coupling system was presented with finite element analysis, Newmark-beta numerical integration method was employed to analyzing the dynamics of the system.

\section{MESHING FORCE OF MISALIGNED SPLINE COUPLING}

\subsection{Deformation of single spline}

Meshing rigidity of gear tooth represents the synthesis effect of all the meshing teeth in the action region $[17,18]$, in case of sufficient lubrication, the friction of tooth surfaces can be neglected, meshing rigidity is mainly correlated with elastic deformation of single gear tooth [19]. Because single spline can be considered as typical tooth, the above theory is also suitable for deriving the spline deformation. The elastic deformation of rectangle spline can be considered as a planar problem, generally. Spline is simplified as a cantilever beam with same section in elastic foundation according to mechanics of materials.

As shown in Figure 1, $f$ is the distribution force acting on the surface of spline, $F_{i}$ is its equivalent concentrate force acting on the meshing point $i$; the deformation caused by $F_{i}$ consists of bending, shear deformation, and deformation of elastic foundation. Because typical spline belongs to width spline $(B / H>5)$, deformation of elastic foundation

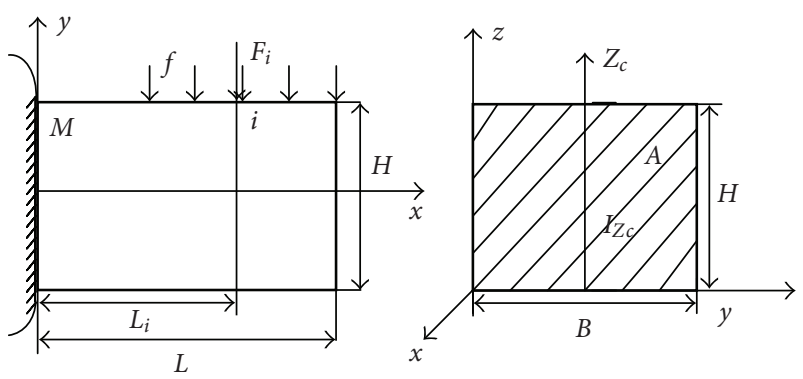

FIgURE 1: Deformation of single spline.

should not be neglected compared to bending and shear deformation.

The area and inertia of cross-section are $A=B H$ and $I_{Z c}=B H^{3} / 12$, respectively; $L_{i}$ is the distance from $i$ to $M$; thickness, width, and height of single spline are $H, B$, and $L$, respectively; spline number is $z$; Poisson's ratio and elastic modulus of spline material are $v$ and $E$, respectively, according to Cornell's analysis [20], for width spline, equivalent elastic modulus $E_{e}$ is $E /\left(1-v^{2}\right)$.

(1) Bending deformation is

$$
\delta_{B i}=\frac{F_{i} L_{i}^{3}}{3 E_{e} I} .
$$

(2) Shear deformation is

$$
\delta_{S i}=\frac{k F_{i} L_{i}}{G A}=\frac{12 F_{i} L_{i}(1+v)}{5 E_{e} A} .
$$

(3) Deformation of elastic foundation, refer to the analysis of gear tooth [20], is

$$
\delta_{M i}=\frac{F_{i}}{E_{e}}\left[\frac{5.306}{B H^{2}} L_{i}^{2}+\frac{2\left(1-v-2 v^{2}\right)}{\left(1-v^{2}\right) B H} L_{i}+\frac{1.534}{B}\right] .
$$

Thus, the total elastic deformation is

$$
\delta_{i}=\delta_{B i}+\delta_{S i}+\delta_{M i}
$$

Therefore, the rigidity of single spline is

$$
\begin{aligned}
K_{i} & =\frac{F_{i}}{\delta} \\
& =\frac{E_{e}}{(1 / 3 I) L_{i}^{3}+\left(5.306 / B H^{2}\right) L_{i}^{2}+[Q+X] L_{i}+1.534 / B} .
\end{aligned}
$$

where $Q=2\left(1-v-2 v^{2}\right) /\left(1-v^{2}\right) B H, X=12(1+v) / 5 A$.

Equation (5) shows that the stiffness of single spline is the function of meshing distance $L_{i}$.

\subsection{Meshing distance of misaligned spline coupling}

When there is no misalignment, the centre of male coupling is superposed with the female, as described in Figure 2, the meshing distance for each spline is the same, and we define it as alignment meshing distance (AMD) with the value of $L_{0}$. 


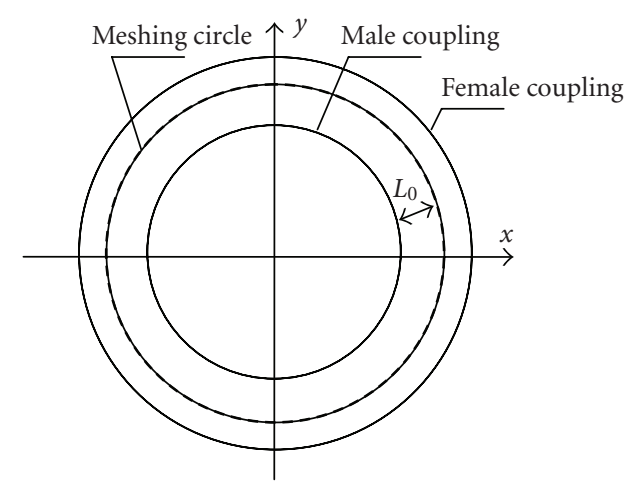

FIGURE 2: AMD of coupling with no misalignment.

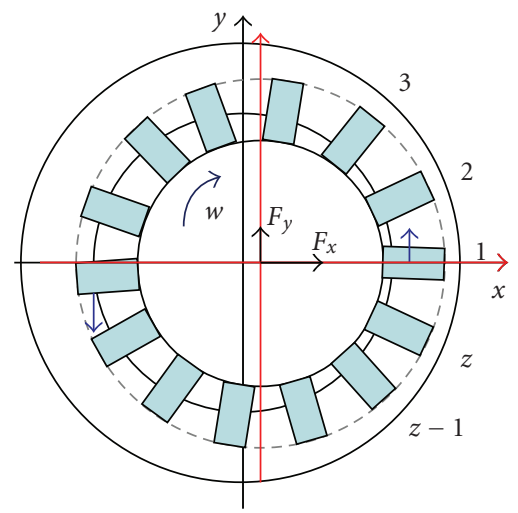

FIGURE 3: Meshing distance of coupling with misalignment in $x$ axis.

For a real system, there are two kinds of misalignments: static misalignment $\left(e_{0}, \varphi_{0}\right)$ and dynamic misalignment (dynamic vibration displacement) $(x, y)$, a general designation of these two is misalignment in the following analysis. So the real misalignment $(e, \varphi)$ is

$$
\begin{gathered}
e=\sqrt{\left(x+e_{0} \cos \varphi_{0}\right)^{2}+\left(y+e_{0} \sin \varphi_{0}\right)^{2}}, \\
\cos \varphi=\frac{x+e_{0} \cos \varphi_{0}}{e}, \\
\sin \varphi=\frac{y+e_{0} \sin \varphi_{0}}{e} .
\end{gathered}
$$

For simplifying, assume that the misalignment takes place in $x$-axis, that is, $\varphi_{0}=0$ and $y=0$. The meshing in two couplings is asymmetric, teeth in right parts of the two couplings meshing tightly, contrarily, the left parts meshing loosely meshing distance for each spline is different as presented in Figure 3.

Assuming the spline serial number is 1 in positive direction of $x$-axis, then it is $2,3, \ldots, z$, anticlockwise, the interangular from each spline to positive direction of $x$-axis is

$$
\varphi_{i}=\frac{2 \pi(i-1)}{z} .
$$

Accordingly, the $L_{i}$ can be presented as

$$
L_{i}=L_{0}-e \cos \varphi_{i} .
$$

With above analysis, meshing rigidity of single spline due to misalignment is

$$
K_{i}=f\left(L_{i}\right)
$$

\subsection{Meshing force of misaligned spline coupling}

In a real rotor-spline coupling system, all splines meshing tightly by transmitting large torque cause a deformation of each spline. At the same time, for coupling nodes vibrating with the system, there is relative displacement between splines in two half-couplings, which cause another deformation of each spline; both the deformations generate meshing force.

(1) Meshing force caused by torque is as follows.

When the torque transmitted by coupling is $T$,

$$
T=\sum_{i=1}^{z}\left[F_{T i} \cdot R_{i}\right]=\sum_{i=1}^{z}\left[F_{T i} \cdot\left(R+L_{i}\right)\right],
$$

$R$ is radius of the circle in spline root, and

$$
F_{T i}=\phi L_{i} \cdot K_{i},
$$

$\phi$ is the torsion angular for each spline caused by deformation, as all splines constitute a whole coupling, $\phi$ 's for all splines are the same when coupling turns around with the static torque $T$. Summarizing the above analysis,

$$
T=\sum_{i=1}^{z}\left[\phi L_{i} \cdot K_{i} \cdot\left(R+L_{i}\right)\right]
$$

So, $\phi$ is

$$
\phi=\frac{T}{\sum_{i=1}^{z}\left[L_{i} K_{i}\left(R+L_{i}\right)\right]} .
$$

Therefore, force transmitted by each spline is

$$
F_{T i}=\phi L_{i} \cdot K_{i} .
$$

(2) Meshing force caused by dynamic vibration displacement is

$$
F_{i}^{\prime}=\left(e^{\prime} \sin \varphi_{\mathrm{i}}\right) \cdot K_{i},
$$

and $e^{\prime}=\sqrt{x^{2}+y^{2}}$.

Accordingly, meshing force caused by misalignment is

$$
F_{i}=F_{T i}+F^{\prime}=\left(\phi L_{i}+e^{\prime} \sin \varphi_{\mathrm{i}}\right) K_{i} .
$$

From above equation, we know that, due to dynamic vibration displacement, meshing forces of splines from 1 to $z / 2$ get larger, others get smaller, and meshing force for each spline would not be negative. Therefore, when the transmitting torque is zero, there would be no meshing 


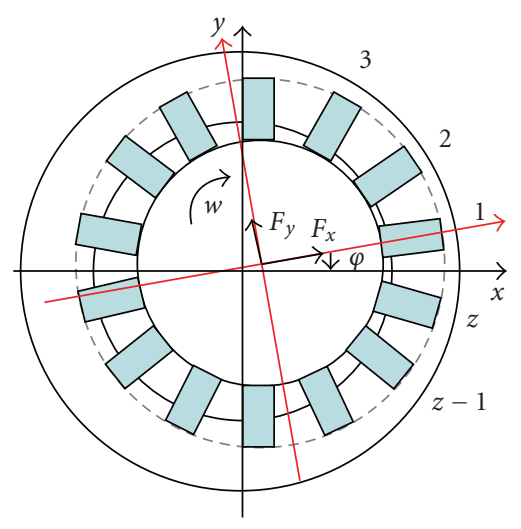

FIGURE 4: Meshing distance of coupling with misalignment in any direction.

force, even dynamic vibration displacement is still present. Therefore,

$$
F_{i}= \begin{cases}\left(\phi L_{i}+e^{\prime} \sin \varphi_{\mathrm{i}}\right) K_{i}, & \phi L_{i}+e^{\prime} \sin \varphi_{\mathrm{i}}>0, \\ 0, & \phi L_{i}+e^{\prime} \sin \varphi_{\mathrm{i}} \leq 0 .\end{cases}
$$

Two components of the above force are

$$
\begin{aligned}
& F_{x i}=F_{i} \cos \theta_{i}, \\
& F_{y i}=F_{i} \sin \theta_{i},
\end{aligned}
$$

$\theta_{i}$ is angular between meshing force and positive direction of $x$-axis, it is

$$
\theta_{i}=\varphi_{i}+\frac{\pi}{2}
$$

The resultant forces due to misaligned coupling are

$$
\begin{aligned}
& F_{x}=\sum_{i=1}^{z} F_{x i}=\sum_{i=1}^{z}\left[\left(\phi L_{i}+e^{\prime} \sin \varphi_{\mathrm{i}}\right) K_{i} \cos \theta_{i}\right], \\
& F_{y}=\sum_{i=1}^{z} F_{y i}=\sum_{i=1}^{z}\left[\left(\phi L_{i}+e^{\prime} \sin \varphi_{\mathrm{i}}\right) K_{i} \sin \theta_{i}\right] .
\end{aligned}
$$

\subsection{Extending of meshing force due to misaligned spline coupling}

The above analysis is a special misalignment that appears in $x$-axis, when the real misalignment is present in any direction, as shown in Figure 4, which means that there is an angular $\varphi$ from $x$-axis, anticlockwise. The definition of $\varphi$ is the same as (6).

The real meshing forces of misaligned coupling are

$$
\begin{gathered}
f_{x}=F_{x} \cos \varphi-F_{y} \sin \varphi, \\
f_{y}=F_{x} \sin \varphi+F_{y} \cos \varphi .
\end{gathered}
$$

From above analysis, the meshing force model of misaligned coupling is achieved; it is a function of coupling parameters, transmitting torque, static misalignment and dynamic vibration displacement, and so on.
TABLE 1: Structure parameters of spline coupling.

\begin{tabular}{lccc}
\hline Name & Symbol & Unit & Value \\
\hline Number of splines & $Z$ & & 14 \\
Spline length & $L$ & $\mathrm{~mm}$ & 5.07 \\
Spline width & $B$ & $\mathrm{~mm}$ & 100 \\
Spline thickness & $H$ & $\mathrm{~mm}$ & 16 \\
AMD (alignment meshing distance) & $L_{0}$ & $\mathrm{~mm}$ & 3.375 \\
Poisson's ratio & $v$ & & 0.3 \\
Elastic modulus & $E$ & $\mathrm{~Pa}$ & $2.12 \times 10^{11}$ \\
Radius of spline root & $R$ & $\mathrm{~mm}$ & 69.5 \\
Transmitting torque & $T$ & $\mathrm{Nm}$ & 87000 \\
\hline
\end{tabular}

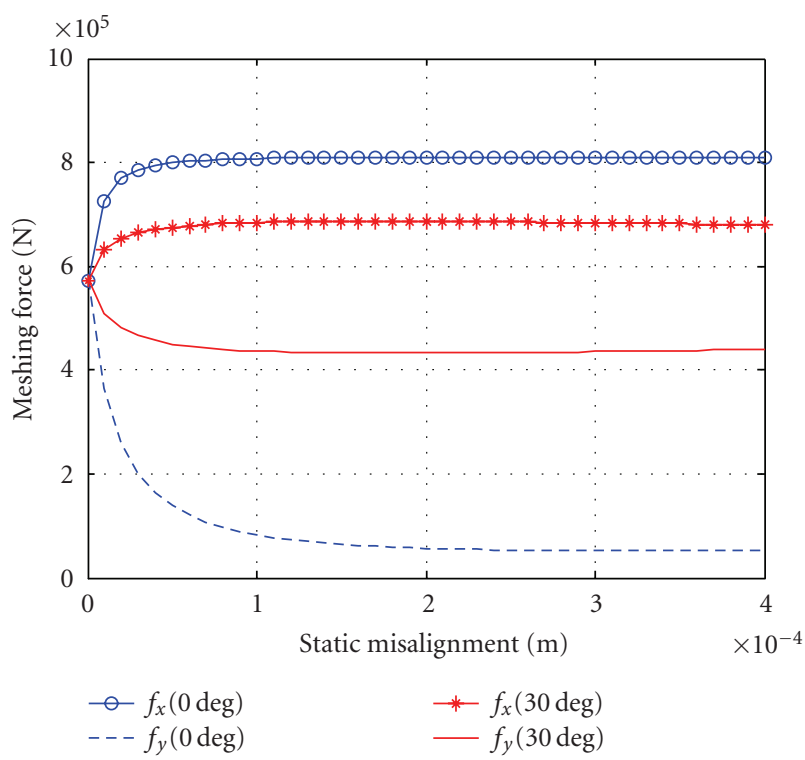

FIGURE 5: Meshing forces versus static misalignment.

\section{SIMULATIONS OF MESHING FORCE}

Structure parameters of spline coupling adopted in this paper are shown in Table 1.

\subsection{Meshing force versus misalignment}

(1) Meshing force versus static misalignment is as follows.

When the dynamic vibration displacement is $x=y=$ $0.001 \mathrm{~mm}$, the static misalignment $e_{0}$ changes from 0 to $1 \mathrm{~mm}$, and $\varphi_{0}$ is $0^{\circ}$ and $30^{\circ}$, respectively, the curves of meshing forces are shown in Figure 5.

According to Figure 5, when the dynamic vibration displacement is small and keeps constant, the following hold.

(a) Two meshing forces are equal when there is no misalignment.

(b) Meshing forces change nonlinearly with smaller static misalignment, while they change linearly when the misalignment gets larger.

(2) Meshing force versus dynamic vibration displacement is as follows. 


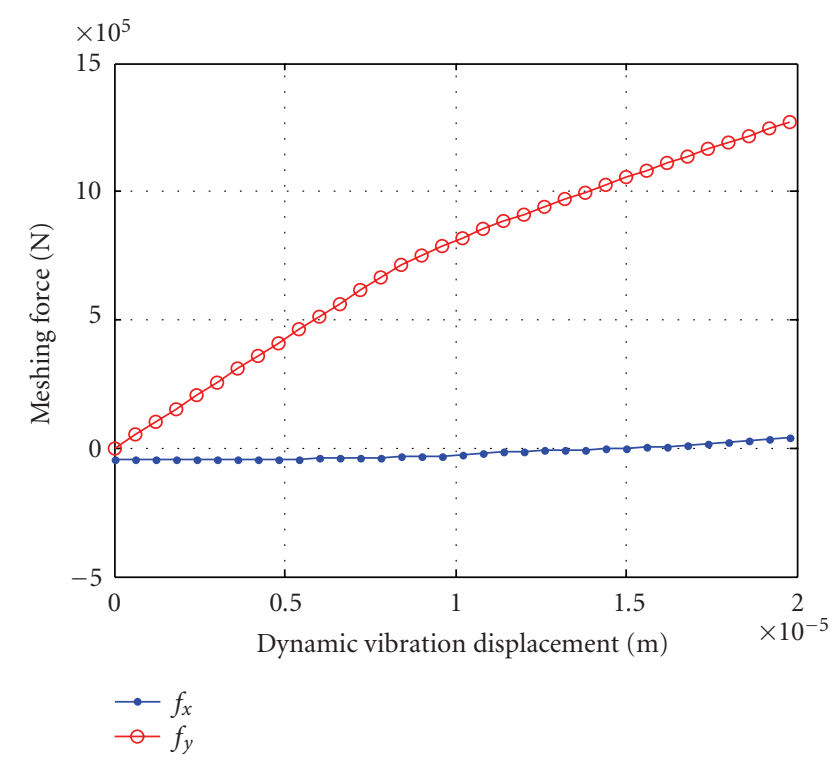

Figure 6: Meshing forces versus dynamic vibration displacement.

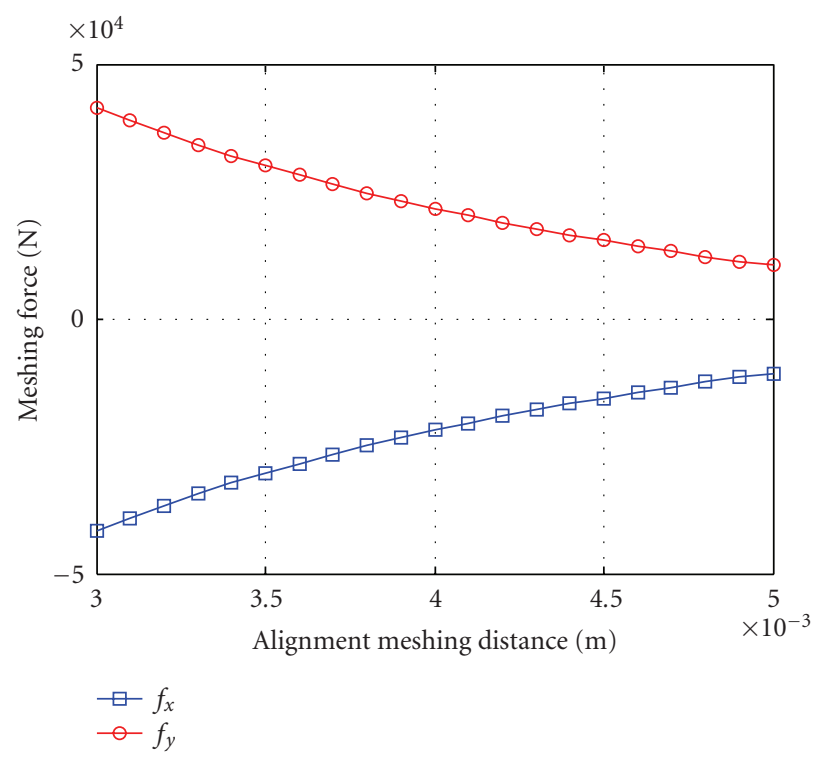

Figure 7: Meshing force versus AMD.

When the static misalignment is $e_{0}=0.5 \mathrm{~mm}, \varphi_{0}=90^{\circ}$, the dynamic vibration displacement of $x$ and $y$ changes from 0 to $0.02 \mathrm{~mm}$, the curves of meshing forces can be obtained as shown in Figure 6.

Figure 6 shows that when the static misalignment appears and keeps constant, the following hold.

(a) Meshing force changes linearly with dynamic vibration displacement, approximately, but there is an inflexion.

(b) The slope coefficient of meshing force curve reflects the stiffness of coupling, consequently, during the vibration of system, the stiffness of coupling is not a constant, it relates to dynamic vibration displacement and depends on static misalignment.

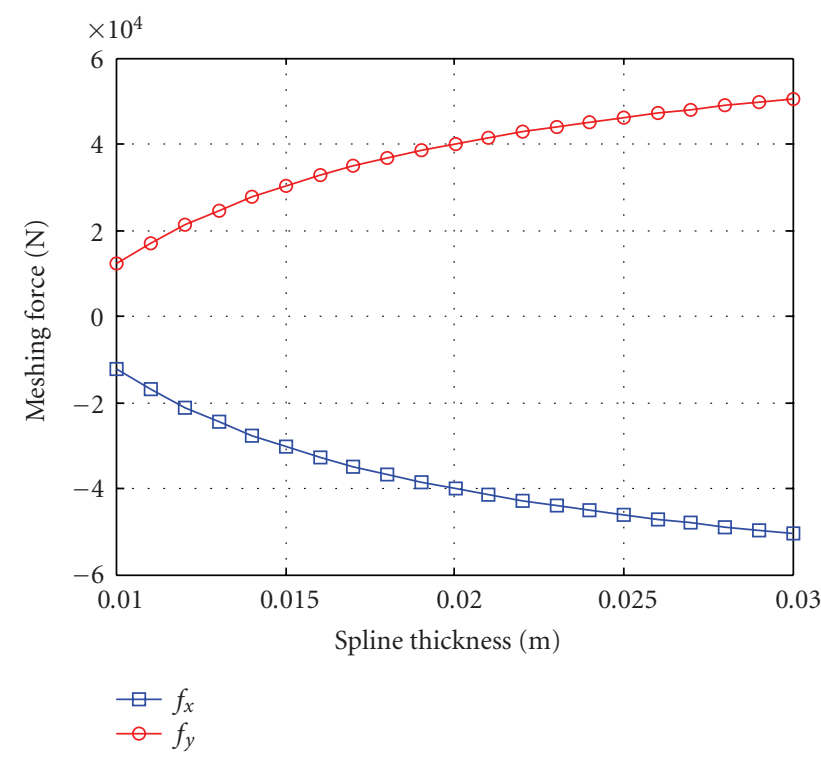

Figure 8: Meshing force versus spline thickness.

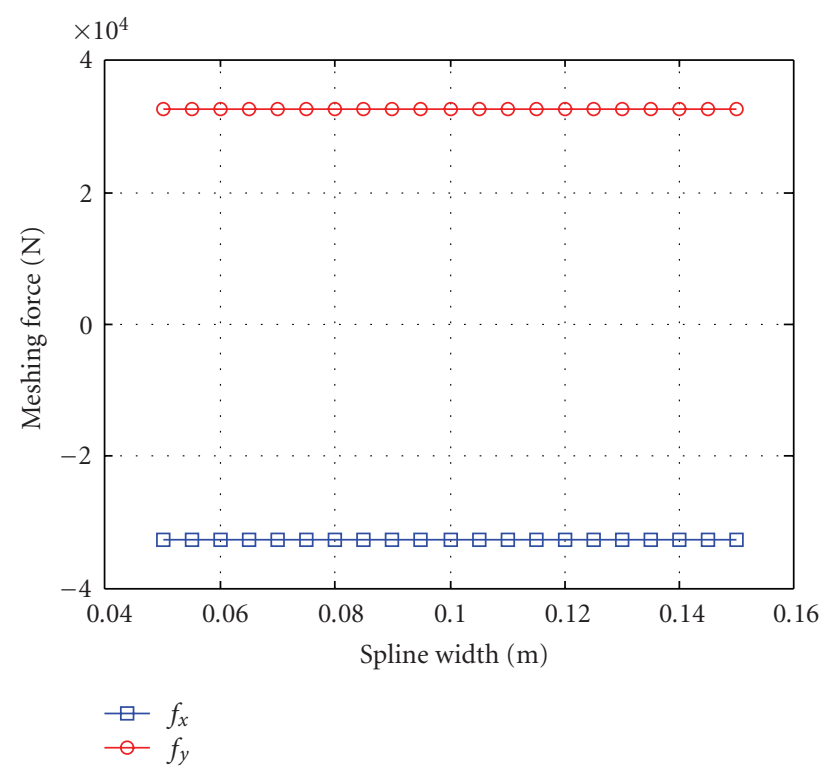

FIGURE 9: Meshing force versus spline width.

\subsection{Meshing force versus coupling parameters}

Choose the misalignment as $x=y=0, e_{0}=0.5 \mathrm{~mm}$, and $\varphi_{0}=45^{\circ}$, change the alignment meshing distance (AMD) $L_{0}$ from 3 to $5.0 \mathrm{~mm}$, the spline thickness $H$ from 10 to $30 \mathrm{~mm}$, and the spline width $B$ from 50 to $150 \mathrm{~mm}$, respectively, the curves of meshing force are represented as shown in Figures 7,8 , and 9 .

The above simulation results show that meshing force increases nonlinearly with the increasing alignment meshing distance (AMD) and with the decreasing spline thickness. The spline number and width have no influence on meshing force directly. However, for a spline coupling, the thickness of spline should be changed when the number of spline alters. 


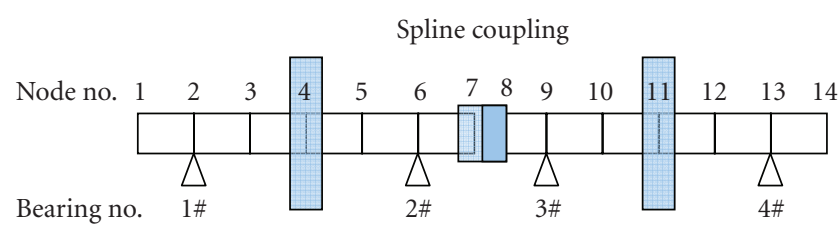

FIGURE 10: Sketch map of rotor-spline coupling system.

\section{DYNAMIC MODEL OF ROTOR-SPLINE COUPLING SYSTEM}

2-span symmetry rotor system was used to study the influence of spline coupling on dyamics of rotor sytem, the sketch map of system is shown in Figure 10.

Based on finite element analysis, presumption of EulerBernoulli beam was employed to derive the dynamic equation of rotor-spline coupling system:

$$
[M]\{\ddot{Z}\}+[C]\{\dot{Z}\}+[K]\{Z\}=\{Q(t)\}+\left\{F_{\mathrm{SP}}\right\} .
$$

For simplifying, the gravity was neglected and the support was assumed as linear spring. In above equation, $[M],[C],[K]$ are system mass, damping (ratio damping), and stiffness matrix, respectively; $\{Q(t)\}$ is the term of unbalance force, $\{Z\}=\left\{x_{j}, y_{j},-\theta_{x j}, \theta_{y j}\right\}$ ( $j$ is the serial number of node), $\left\{F_{\mathrm{SP}}\right\}$ is the meshing force of spline coupling, it is

$$
\begin{aligned}
& f_{x}=F_{x}\left(e, e^{\prime}\right) \cos \varphi-F_{y}\left(e, e^{\prime}\right) \sin \varphi, \\
& f_{y}=F_{x}\left(e, e^{\prime}\right) \sin \varphi+F_{y}\left(e, e^{\prime}\right) \cos \varphi .
\end{aligned}
$$

In above equation,

$$
\begin{gathered}
e=\sqrt{\left(e_{0} \cos \varphi_{0}+d x\right)^{2}+\left(e_{0} \sin \varphi_{0}+d y\right)^{2}}, \\
e^{\prime}=\sqrt{(d x)^{2}+(d y)^{2}}, \\
\cos \varphi=\frac{e_{0} \cos \varphi_{0}+d x}{e}, \\
\sin \varphi=\frac{e_{0} \sin \varphi_{0}+d y}{e},
\end{gathered}
$$

$d x$ and $d y$ are relative displacements of two half-coupling nodes.

\section{DYNAMICS ANALYSIS OF ROTOR-SPLINE COUPLING SYSTEM}

Nowadays, the effective method to analyze (22) is numerical method. Newmark-beta stepping integration method which has high efficiency and good stability was employed to solve the response of system in this paper.

Choosing the static misalignment $\left(e_{0}, \varphi_{0}\right)$ as $(0,0)$, $\left(0.4 \mathrm{~mm}, 45^{\circ}\right)$, and $\left(0.8 \mathrm{~mm}, 45^{\circ}\right)$, respectively, the responses of node 7 (a half-coupling located) in the speed of $5000 \mathrm{rpm}$

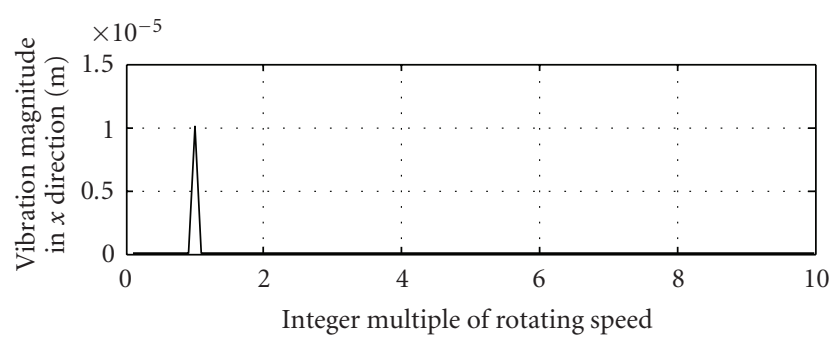

(a)

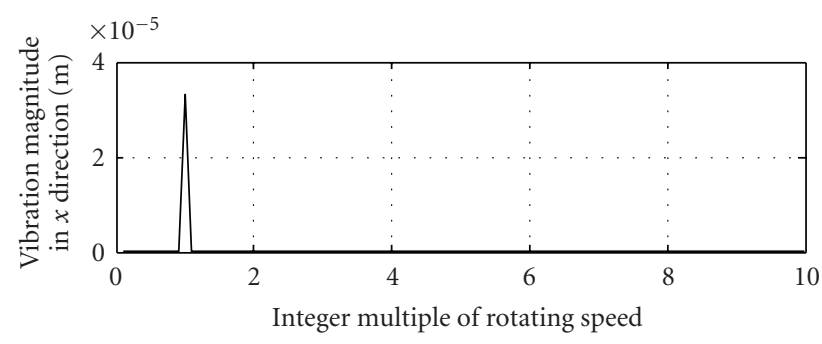

(b)

Figure 11: Frequency $(0,0)$.

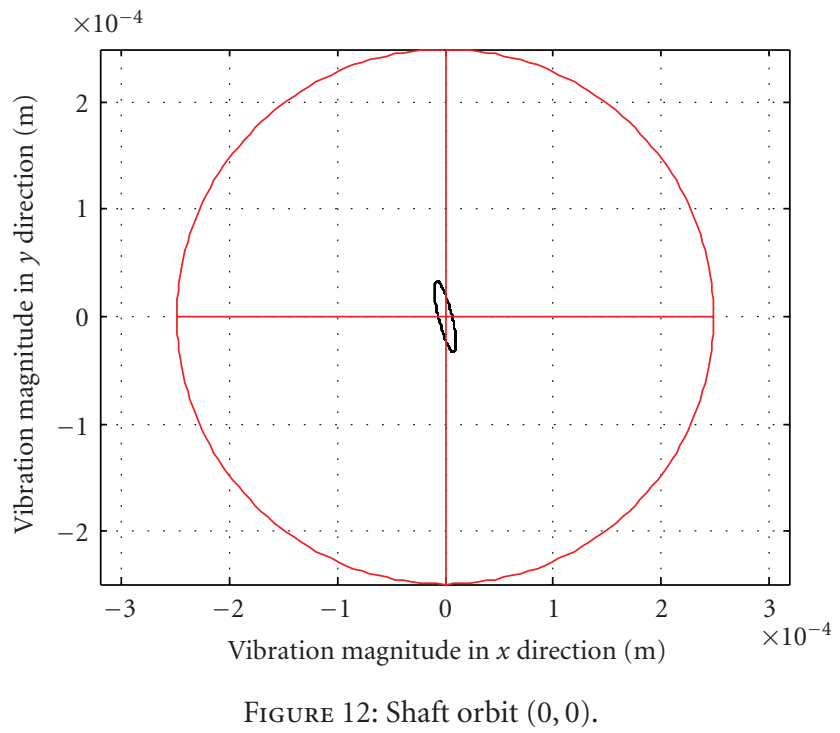

are shown in Figures 11 and 12, 13 and 14, 15, and 16, respectively.

From above analysis, some conclusions can be achieved as follows.

(1) When there is no misalignment, 1X-rotating speed is the main response frequency; shaft orbit encloses origin.

(2) When there is misalignment, 1X- and 2X-rotating speed are the main response frequencies.

(3) With appearance and increases of misalignment, shaft orbit departs from origin, magnitudes of all frequencies increase, and frequency of $2 \mathrm{X}$-rotating speed increases most rapidly. 


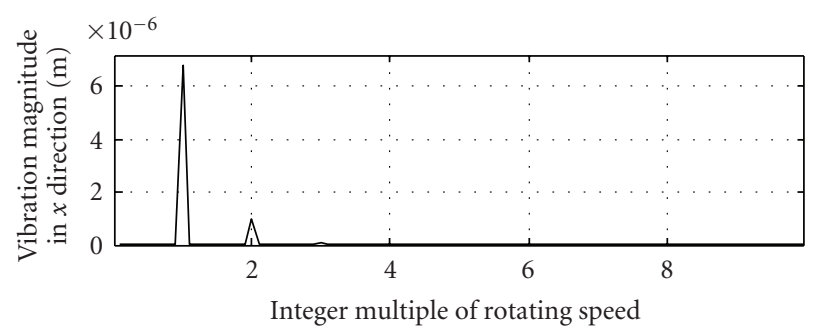

(a)

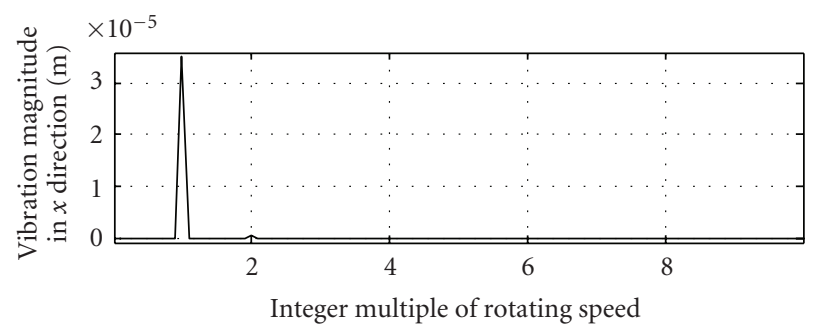

(b)

Figure 13: Frequency $\left(0.4 \mathrm{~mm}, 45^{\circ}\right)$.

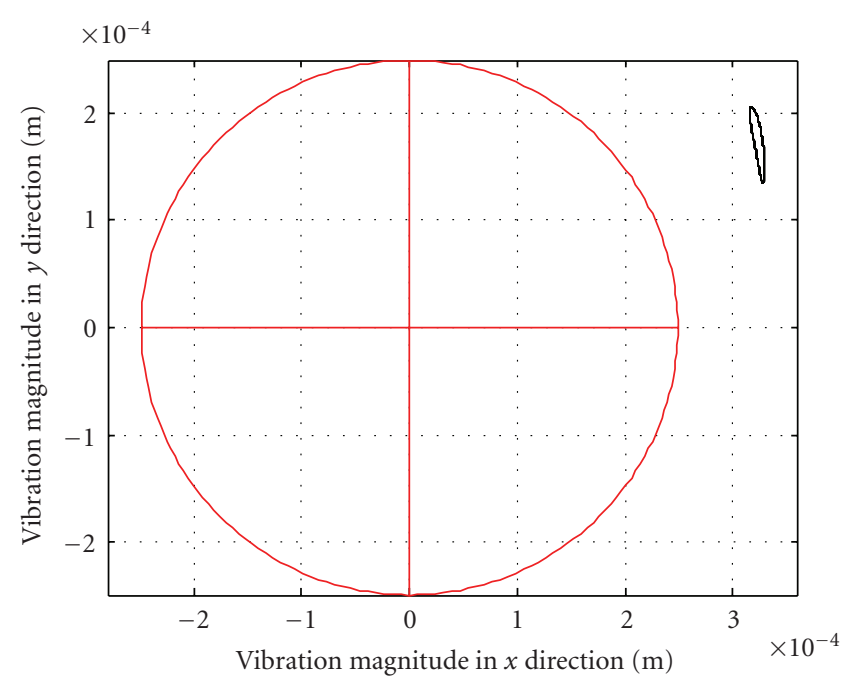

Figure 14: Shaft orbit $\left(0.4 \mathrm{~mm}, 45^{\circ}\right)$.

\section{CONCLUSIONS}

In this study, meshing force of misaligned spline coupling and its influence on rotor system are studied. The followings are the outcome of the study.

(1) Meshing force of misaligned spline coupling is not related to spline number and width directly, it changes linearly with transmitting torque while nonlinearly with alignment meshing distance (AMD), spline thickness, and static misalignment.

(2) Stiffness of coupling is not a constant; it relates to dynamic vibration displacement and depends on static misalignment.

(3) The dynamics of rotor-spline coupling system shows the following: 1X-rotating speed is the main response frequency when there is no misalignment; while $2 \mathrm{X}$-rotating

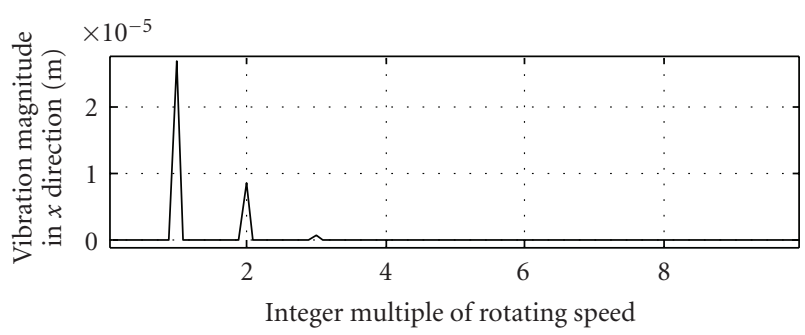

(a)

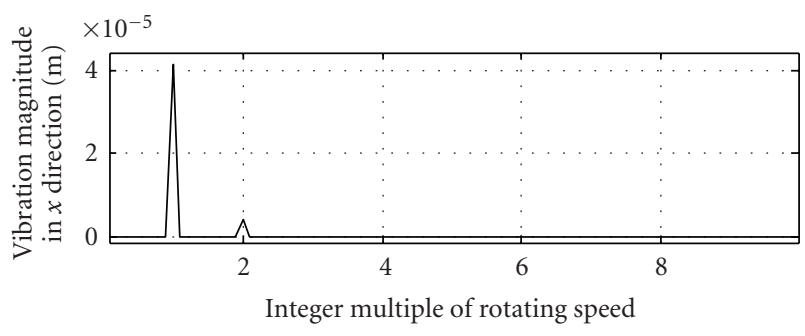

(b)

Figure 15: Frequency $\left(0.8 \mathrm{~mm}, 45^{\circ}\right)$.

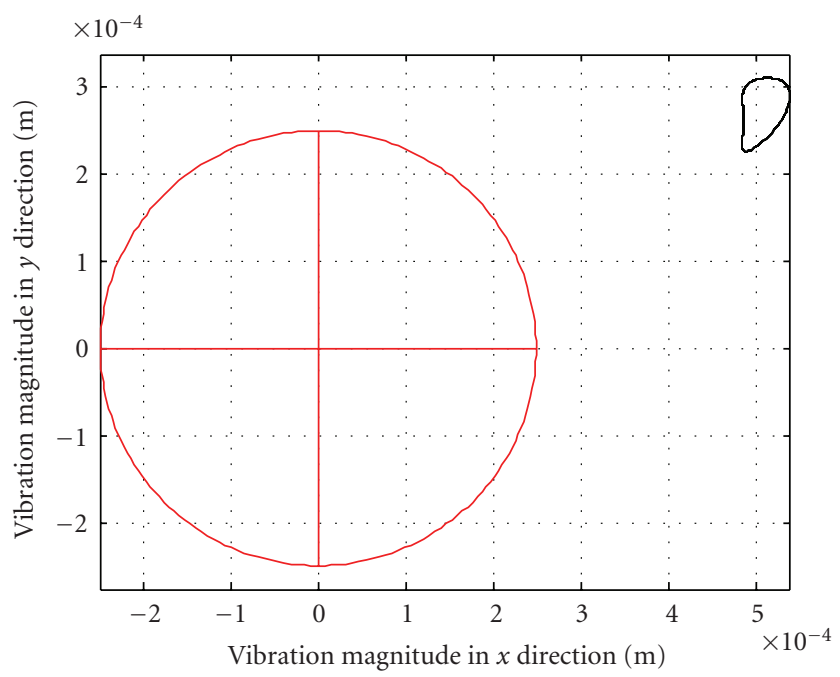

FIgURe 16: Shaft orbit $\left(0.8 \mathrm{~mm}, 45^{\circ}\right)$.

speed appears when misalignment is present; with the increase of misalignment, shaft orbit departs from origin, the magnitudes of all frequencies increase, and 2X-rotating speed increases rapidly. Thus, misalignment makes the vibration of system more complicated.

(4) In order to actualize optimization design of spline coupling with the purpose of reducing the meshing force caused by misalignment, reducing the transmitting torque and misalignment are effectual ways. Moreover, decreasing spline thickness (the number of splines should increase homologically) or increasing the alignment meshing distance (AMD) is also suitable.

(5) During the trouble shooting to rotor-spline coupling system with the aim of decreasing the rotor vibration and reducing frequency components, the effective operations are aligning the support, monitoring the shaft orbit, and 
observing the magnitudes of all frequencies especially the 2X-rotating speed which is considered to be a characteristic commonly observed in the field of misaligned rotating machinery.

\section{ACKNOWLEDGMENT}

This work was supported by the National Nature Science Foundation of China under Grant no. 10632040.

\section{REFERENCES}

[1] K. M. Al-Hussain and I. Redmond, "Dynamic response of two rotors connected by rigid mechanical coupling with parallel misalignment," Journal of Sound and Vibration, vol. 249, no. 3, pp. 483-498, 2002.

[2] S. Medina and A. V. Olver, "Regimes of contact in spline couplings," Journal of Tribology, vol. 124, no. 2, pp. 351-357, 2002.

[3] Q.-H. Li, "Analysis of distance between keys in involute spline coupling of aviation generates," Aviation Precision Manufacturing Technology, vol. 40, no. 2, pp. 40-42, 2004, (Chinese).

[4] K. M. Al-Hussain, "Dynamic stability of two rigid rotors connected by a flexible coupling with angular misalignment," Journal of Sound and Vibration, vol. 266, no. 2, pp. 217-234, 2003.

[5] W. D. Weatherford and M. L. Valtierra, "Mechanisms of wear in misaligned splines," ASME Journal of Lubrication Technology, vol. 90F, pp. 42-48, 1968.

[6] R. A. Newley, The mechanisms of fretting wear of misaligned splines in the presence of a lubricant, Ph.D. thesis, University of London, London, UK, 1978.

[7] J. Han and L.-D. Shi, "Study on kinematic mechanism of misalignment fault of rotor system connected by gear coupling," Journal of Vibration Engineering, vol. 17, no. 4, pp. 416-420, 2004, (Chinese).

[8] H. C. Qu, "The modeling and theoretical analysis of the misalignment default of shaft system in the rotating machinery," Journal of Civil Aviation University of China, vol. 17, no. 3, pp. 11-16, 1999, (Chinese).

[9] A. S. Sekhar and B. S. Prabhu, "Effects of coupling misalignment on vibrations of rotating machinery," Journal of Sound and Vibration, vol. 185, no. 4, pp. 655-671, 1995.

[10] R. A. Marmol, A. J. Smalley, and J. A. Tecza, "Spline coupling induced nonsynchronous rotor vibrations," ASME Journal of Mechanical Design, vol. 102, no. 1, pp. 168-176, 1980.

[11] S. K. Park, Determination of loose spline coupling coefficients of rotor bearing systems in turbomachinery, Ph.D. thesis, Texas A\&M University, College Station, Tex, USA, 1991.

[12] R. H. Bannister, "Methods for modeling flanged and curvic couplings for dynamic analysis of complex rotor constructions," ASME Journal of Mechanical Design, vol. 102, no. 1, pp. 130-139, 1980.

[13] C.-P. R. Ku, J. F. Walton Jr., and J. W. Lund, "An investigation of angular stiffness and damping coefficients of an axial spline coupling in high-speed rotating machinery," NASA Technical Reports Server (NTRS) N94-34192, pp. 293-303, NASA Center, Washington, DC, USA, 1994.

[14] C.-P. R. Ku, J. F. Walton, and J. W. Lund, "Dynamic coefficients of axial spline couplings in high-speed rotating machinery," ASME Journal of Vibration and Acoustics, vol. 116, no. 3, pp. 250-256, 1994.
[15] H.-P. Peng and Z.-M. Li, "A research on the numerical relations between the load capacity and making precision of spline on the ends of torsion bar spring," Mechanism, vol. 31, no. 5, pp. 20-25, 2004, (Chinese).

[16] J. F. Walton Jr., A. Artiles, and J. W. Lund, "Internal rotor friction instability," MTI 88TR39, Mechanical Technology Incorporated, Latham, NY, USA, 1990.

[17] M. S Tavikoli and D. R. Houser, "Optimum profile modification for the minimization of static transmission errors of spur gear," ASME Journal of Mechanisms, Transmissions, and Automation in Design, vol. 108, pp. 86-94, 1986.

[18] C.-J. Zhou, J.-Y. Tang, and Y.-X. Wu, "The comparative study of the bending stress and elastic deformation calculation of gear tooth," Journal of Mechanical Transmission, vol. 28, no. 5, pp. 1-6, 2004, (Chinese).

[19] Z.-S. Liu and G. Zhao, "Modeling research on radial force in gear coupling with parallel misalignment," in Proceedings of the 12th World Congress in Mechanism and Machine Science (IFToMM '07), BesanÇon, France, June, 2007, A764.

[20] R. W. Cornell, "Compliance and stress sensitivity of spur gear teeth," ASME Journal of Mechanical Design, vol. 103, no. 2, pp. 447-459, 1981. 

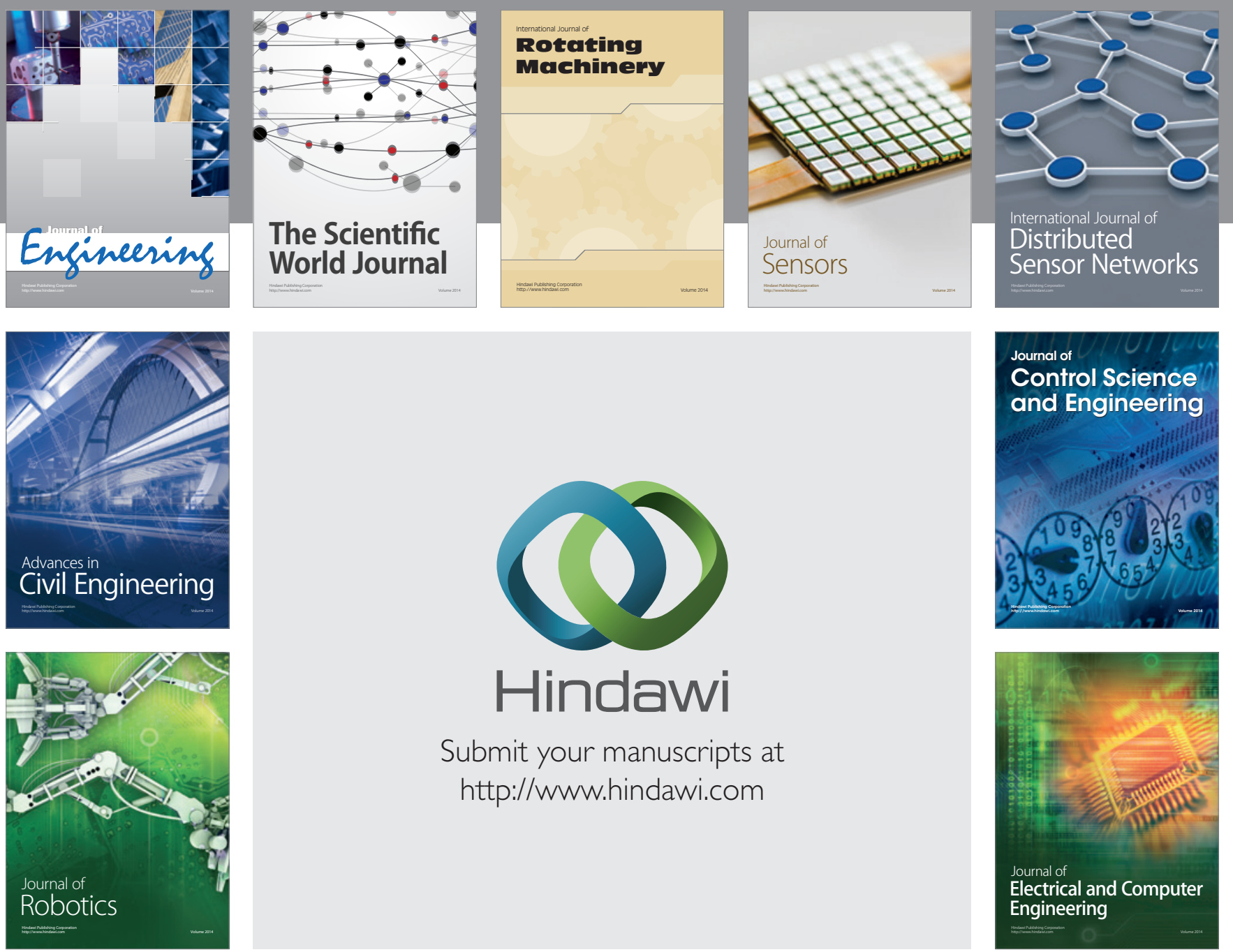

Submit your manuscripts at

http://www.hindawi.com
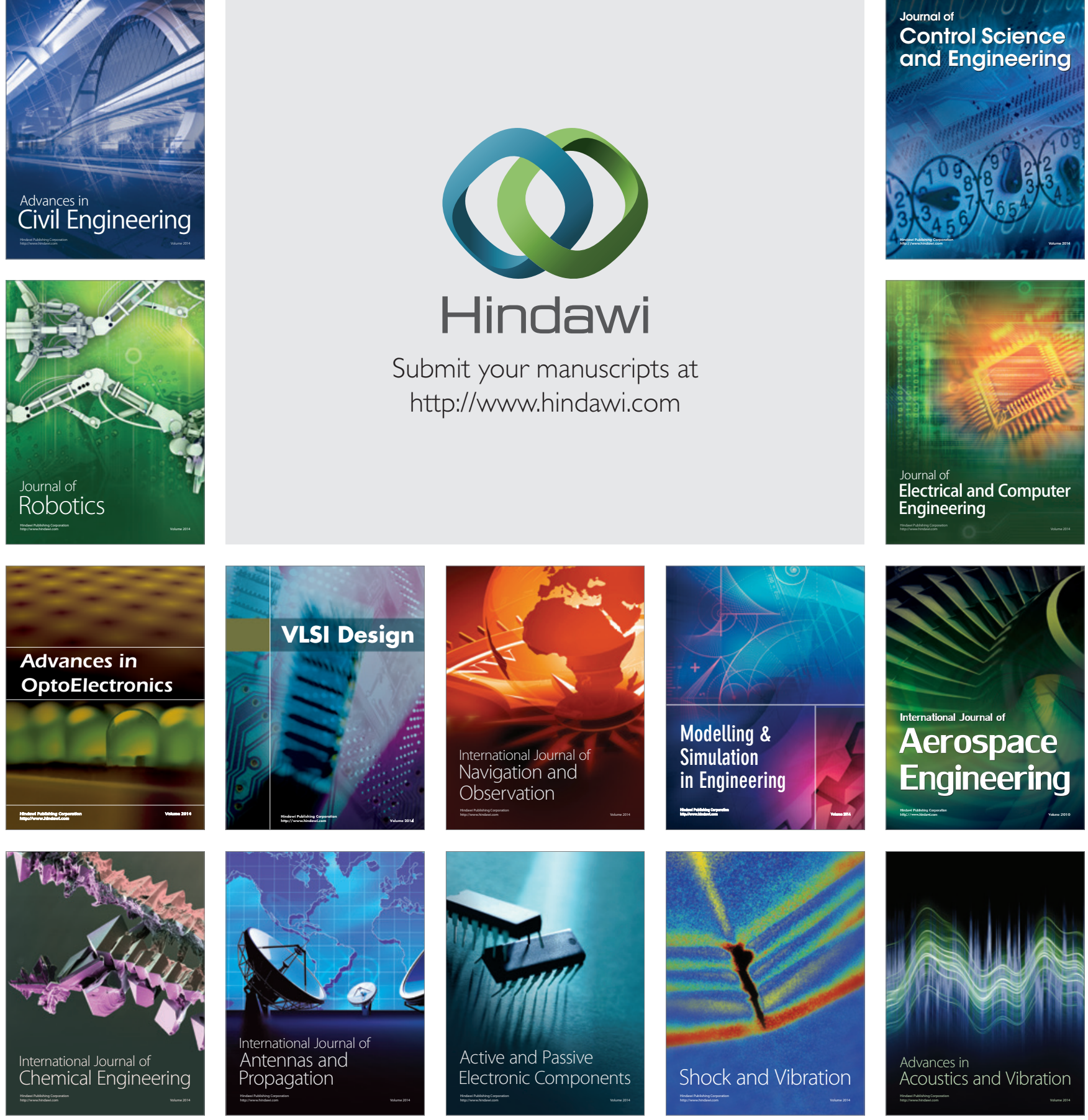